\title{
BMJ Open Feasibility and acceptability of personalised breast cancer screening (DECIDO study): protocol of a single- arm proof-of-concept trial
}

Anna Pons-Rodriguez, ${ }^{1,2}$ Carles Forné Izquierdo (1) , ,3 Jordi Vilaplana-Mayoral, ${ }^{5}$ Inés Cruz-Esteve, ${ }^{6}$ Isabel Sánchez-López, ${ }^{7}$ Mercè Reñé-Reñé, ${ }^{8}$ Cristina Cazorla, ${ }^{6}$ Marta Hernández-Andreu, ${ }^{6}$ Gisela Galindo-Ortego, ${ }^{6}$ Montserrat Llorens Gabandé, ${ }^{9}$ Celmira Laza-Vásquez, ${ }^{2}$ Pau Balaguer-Llaquet, ${ }^{10}$ Montserrat Martínez-Alonso, ${ }^{3,4,10}$ Montserrat Rué (iD , 3,4,10 The DECIDO Group

To cite: Pons-Rodriguez A, Forné Izquierdo C, VilaplanaMayoral J, et al. Feasibility and acceptability of personalised breast cancer screening (DECIDO study): protocol of a single-arm proof-ofconcept trial. BMJ Open 2020;10:e044597. doi:10.1136/ bmjopen-2020-044597

- Prepublication history for this paper is available online. To view these files, please visit the journal online (http://dx.doi. org/10.1136/bmjopen-2020044597).

MM-A and MR contributed equally.

Received 09 September 2020 Revised 28 November 2020 Accepted 08 December 2020

Check for updates

(c) Author(s) (or their employer(s)) 2020. Re-use permitted under CC BY-NC. No commercial re-use. See rights and permissions. Published by BMJ.

For numbered affiliations see end of article.

Correspondence to Professor Montserrat Rué; montserrat.rue@udl.cat

\section{ABSTRACT}

Introduction Personalised cancer screening aims to improve benefits, reduce harms and being more costeffective than age-based screening. The objective of the DECIDO study is to assess the acceptability and feasibility of offering risk-based personalised breast cancer screening and its integration in regular clinical practice in a National Health System setting.

Methods and analysis The study is designed as a single-arm proof-of-concept trial. The study sample will include 385 women aged $40-50$ years resident in a primary care health area in Spain. The study intervention consists of (1) a baseline visit; (2) breast cancer risk estimation; (3) a second visit for risk communication and screening recommendations based on breast cancer risk and (4) a follow-up to obtain the study outcomes.

A polygenic risk score (PRS) will be constructed as a composite likelihood ratio of 83 single nucleotide polymorphisms. The Breast Cancer Surveillance Consortium risk model, including age, race/ethnicity, family history of breast cancer, benign breast disease and breast density will be used to estimate a preliminary 5-year absolute risk of breast cancer. A Bayesian approach will be used to update this risk with the PRS value.

The primary outcome measures will be attitude towards, intention to participate in and satisfaction with personalised breast cancer screening. Secondary outcomes will include the proportions of women who accept to participate and who complete the different phases of the study. The exact binomial and the Student's t-test will be used to obtain $95 \%$ Cls.

Ethics and dissemination The study protocol was approved by the Drug Research Ethics Committee of the University Hospital Arnau de Vilanova. The trial will be conducted in compliance with this study protocol, the Declaration of Helsinki and Good Clinical Practice.

The results will be published in peer-reviewed scientific journals and disseminated in scientific conferences and media.

Trial registration number NCT03791008.

\section{Strengths and limitations of this study}

- This proof-of-concept study will provide evidence on feasibility and acceptance of personalised breast cancer screening, by the target population, under a publicly funded health system.

- Breast cancer risk will be estimated using known risk factors and a polygenic risk score.

- Tailored recommendations on breast cancer screening will be provided to women by primary care doctors in their health centre.

- Women's attitudes towards, participating intentions in and satisfaction with personalised breast screening will be assessed as primary outcomes.

- The design of a single arm has some limitations, such as having no outcome comparisons between groups.

\section{INTRODUCTION}

The Horizon 2020 Advisory Group defines personalised medicine as 'a medical model using characterisation of individuals' phenotypes and genotypes for tailoring the right therapeutic or preventive strategy for the right person at the right time'. ${ }^{1}$ Personalised cancer screening pursues improving age-based screening by detecting malignant tumours in younger subjects at higher risk, reducing harms through reduction of falsepositive results and overdiagnosis in subjects at lower risk and being more cost-effective. ${ }^{23}$

Personalised screening for breast cancer consists of estimating the individual risk of developing breast cancer in a specific time horizon and providing tailored recommendations for early detection that combine (1) frequency of the screening exams (eg, annual, biennial, triennial); (2) age at the 
start and end of screening and (3) screening modality (mammogram, ultrasound, MRI).

Personalised screening requires an accurate measure of individual risk. Tice $e t a l^{4}$ developed and validated the Breast Cancer Surveillance Consortium (BCSC) model including age, race or ethnicity, breast density, family history of breast cancer and previous biopsy. An updated version of the BCSC model replaced previous biopsy by type of benign breast disease. ${ }^{5}$ Recently, genome studies have identified $>90$ genetic variants called singlenucleotide polymorphisms (SNPs) that would explain between $15 \%$ and $20 \%$ of the inherited variance in breast cancer risk. ${ }^{6}$ Michailidou $e t a l^{7}$ identified 65 new breast cancer risk loci and estimated that, in total, common susceptibility variants explain $18 \%$ of the familial relative risk. Mavaddat $e t a l^{8}$ proposed a polygenic risk score (PRS) that grouped the individual effects and the interactions of 77 SNPs to estimate the risk of breast cancer. Vachon $e t a t^{10}$ observed independence between a PRS similar to the previous one and breast density in casecontrol studies.

In a critical review of clinical applications of polygenic breast cancer risk, Yanes $e t a l^{11}$ highlight that there is considerable debate about the clinical utility of polygenic information for assessing breast cancer risk. Whereas, the sceptics argue that there is not enough evidence for their implementation in clinical practice, the supporters consider that PRS have the potential of (1) providing risk information to women with uninformative genetic testing results; (2) being risk modifiers for those with pathogenic variants in high and moderate risk genes and (3) providing personalised risk assessments and risk management strategies in population screening programmes. In fact, the Yanes et al review ${ }^{11}$ showed that, in European populations, the addition of a PRS to the existing risk models has improved their accuracy. The highest area under the curve, 0.72 , was reported in a model that combined BCSC, circulating oestradiol levels and PRS, for oestrogen receptor-positive breast cancer. ${ }^{12}$

Few studies have assessed the implementation of riskbased breast cancer screening. Román et $a l^{13}$ performed the first systematic review of studies assessing personalised breast cancer screening strategies and evaluated the quality of the evidence. Thirteen studies were included, three randomised controlled trials in the recruitment phase and no reported results yet, nine mathematical modelling studies and one observational pilot study. In all models and in the observational study, personalised screening strategies were shown to be effective and efficient. However, as the authors indicate, evidence is lacking on feasibility, acceptability and the legal and ethical aspects of personalised screening strategies.

Evans $e t a l^{14}$ assessed the feasibility of determining breast cancer risk and communicating it in the context of a population-based mammographic screening programme in England. In the Predicting Risk of Cancer At Screening (PROCAS) study, they used the Tyrer-Cuzick model ${ }^{15}$ for risk assessment and investigated risk perception, the proportion wishing to know their 10-year risk and whether subsequent screening attendance was affected. Women at high or at low risk were invited for risk feedback and counselling. The authors concluded that (a) the majority of women wished to receive risk information; (b) perception of breast cancer risk in the general population is poor and (c) high-risk women increased their attendance to the subsequent screening. In a later analysis, Evans et $a l^{16}$ added a PRS and mammographic density to the TyrerCuzick model, with the objective of improving breast cancer risk stratification and enabling more targeted early detection/prevention strategies in population screening programmes. The combined risk tool improved the Tyrer-Cuzick model and defined a low-risk group of women that was approximately $30 \%$ of the total, such that cancers identified in this group were significantly more likely to have an extremely good prognosis. According to the authors, in a risk-stratified approach with an assessment at first mammogram around age $45-50$ years, the extra screening in the high-risk group could be offset by reducing or eliminating screening in the low-risk group, where the benefits of screening may be outweighed by false-positive results and the potential for overdiagnosis and overtreatment. ${ }^{16}$

On the basis of the PROCAS project, and considering the National Institute for Health and Care Excellence clinical guidelines for women with familial breast cancer, French et $a l^{17}$ developed an automated system (BC-Predict) for offering an assessment of breast cancer risk to women when they receive their National Health Service Breast Screening Programme (NHSBSP) invitation, and generating feedback letters to communicate this risk to women and health professionals. The recently published protocol describes that the study aims at identifying and resolving key uncertainties regarding the feasibility of integrating BC-Predict into the NHSBSP. ${ }^{17}$ In addition to including an explicit quantitative and qualitative analysis of the effects of implementing the BC-Predict system in the NHSBSP, the research will assess the feasibility of a definitive study to evaluate whether the intervention translates into measurable effects on breast cancer incidence and stage, and is a cost-effective use of the National Health Service resources.

In Spain, most of population-based screening programmes target women aged 50-69 years and perform biennial mammograms. Extending the programme to older or younger women has been a matter of interest for health policy-makers. On the one hand, opportunistic screening in women younger than 50 years is widely used. The InforMa study found that around $80 \%$ of the participants reported previous use of screening mammograms before age 50 years, outside the organised screening programme, ${ }^{18}$ with potential harms of screening such as overdiagnosis and false-positive results in low-risk women. On the other hand, in Catalonia, the incidence of ductal carcinoma in situ and invasive breast cancer in young women increased steadily during the 1990s and the first decade of the 2000s, in parallel with an increase in 
mammography use over time in women in their late $30 \mathrm{~s}$ or early 40 s. ${ }^{19}{ }^{20}$ Including women younger than 50 years in risk-based screening, so low-risk women are recommended to wait and high-risk women are screened, may improve the balance of benefits and harms of the intervention, as mathematical models have shown. ${ }^{3}$

There will be several challenges to the implementation of personalised screening. In women, barriers related to their culture, socioeconomic level, personal experience, the trust in the infallibility and innocuousness of screening and the social alarm that cancer generates, among others. ${ }^{21} 22$ In healthcare professionals, some barriers are common with those in women and others are specific, such as the inertia to adapt to new evidence and the social pressure to avoid clinical error or delayed diagnosis. ${ }^{23-25}$ Two systematic reviews showed that both women and healthcare professionals overestimate benefits and underestimate harms of treatments, screening and tests. ${ }^{26}{ }^{27}$ In addition, women overestimate the risk of breast cancer and most of them have not been informed of the adverse effects of screening. In clinical practice, personalised screening probably will require more time than the current 'one-size-fits-all' approach, at least at the beginning. But new ways of organising, integrating and analysing data will allow for better identification of individual features that could indicate the optimal intervention, at any time, while saving time and increasing effectiveness and efficiency. When the ongoing clinical trials finish, if they show that risk-based screening is effective and efficient with respect to the current practice, country-specific proof-of-concept studies on implementing the risk-based screening approach will be of great value.

\section{Study objective}

The objective of the DECIDO project is to assess the acceptability and feasibility of offering personalised breast cancer screening and its integration in the usual clinical practice. This proof-of-concept study will assess the women's attitudes, participating intentions and satisfaction with personalised breast screening.

\section{METHODS AND ANALYSIS \\ Study design}

The study is designed as a single-arm proof-of-concept trial. A pilot study was carried out with 20 women to test the suitability of the recruitment and data collection processes and the coordination of the involved healthcare professionals.

\section{Participants}

From January 2019 onwards, 385 women aged 40-50 years will be enrolled in the study. Potential participants are the 2038 women living in the 'Primer de Maig' Basic Health Area in Lleida, Catalonia, on 31 December 2018, that would turn 40-50 years during the following 1.5 years, according to the primary care information system.
All the women that will turn 50 during the study period will be invited to participate. If they decline to participate in the study, they will be invited to participate in the population-based Early Detection Programme for Breast Cancer, since women resident in Spain, aged 50-69 years, are invited biennially for a breast screening mammogram.

From women that will turn 40-49 years during the study period, a random sample of 20-50 women will be selected, on a monthly basis, and invited to participate until the accrual goal of 385 women is achieved.

Women with a previous diagnosis of breast cancer or a breast study in process, or those fulfilling clinical criteria for cancer genetic counselling will be excluded. Women not understanding or speaking Catalan or Spanish, or with a physical or cognitive disability that prevents breast screening or the main outcomes assessment will be also excluded.

An invitation letter with a brief summary of the study objectives will be mailed to the selected women. After 1-2 weeks, a primary care doctor of the study team will make a phone call, provide a brief information about the study, determine eligibility and ask if they are interested in participating. Women that will to participate will be scheduled for a visit at the primary care centre. An informed consent form will be obtained at the beginning of the first visit.

\section{Intervention}

The study intervention consists of a baseline visit, the breast cancer risk estimation, a visit for risk communication and screening recommendations and the administration of a follow-up questionnaire. Figure 1 shows the timeline of the intervention.

\section{First visit at the primary care centre}

The study intervention consists of: (1) providing detailed information about the study objectives by a primary care doctor, member of the study team; (2) providing an informative brochure about the benefits and adverse effects of breast cancer screening ${ }^{28}$; (3) obtaining information on sociodemographic variables, breast cancer risk factors, previous screening experience, perceived personal risk of breast cancer and general screening knowledge, attitudes and intentions; (4) obtaining a saliva sample to determine the genomic profile and (5) scheduling a screening mammogram with breast density measurement. For women that had a mammogram during the year before the first visit, breast density and presence/absence of benign lesions will be obtained from that mammogram and the radiologist report.

\section{Breast density measurement and breast findings}

A senior radiologist (MR-R) expert in screening will evaluate the mammograms of all participating women. Mammographic breast density will be classified according to the Breast Imaging Reporting and Data System (BIRADS) scoring system ${ }^{29}$ : almost entirely fatty (a), scattered areas of fibroglandular density (b), heterogeneously dense 


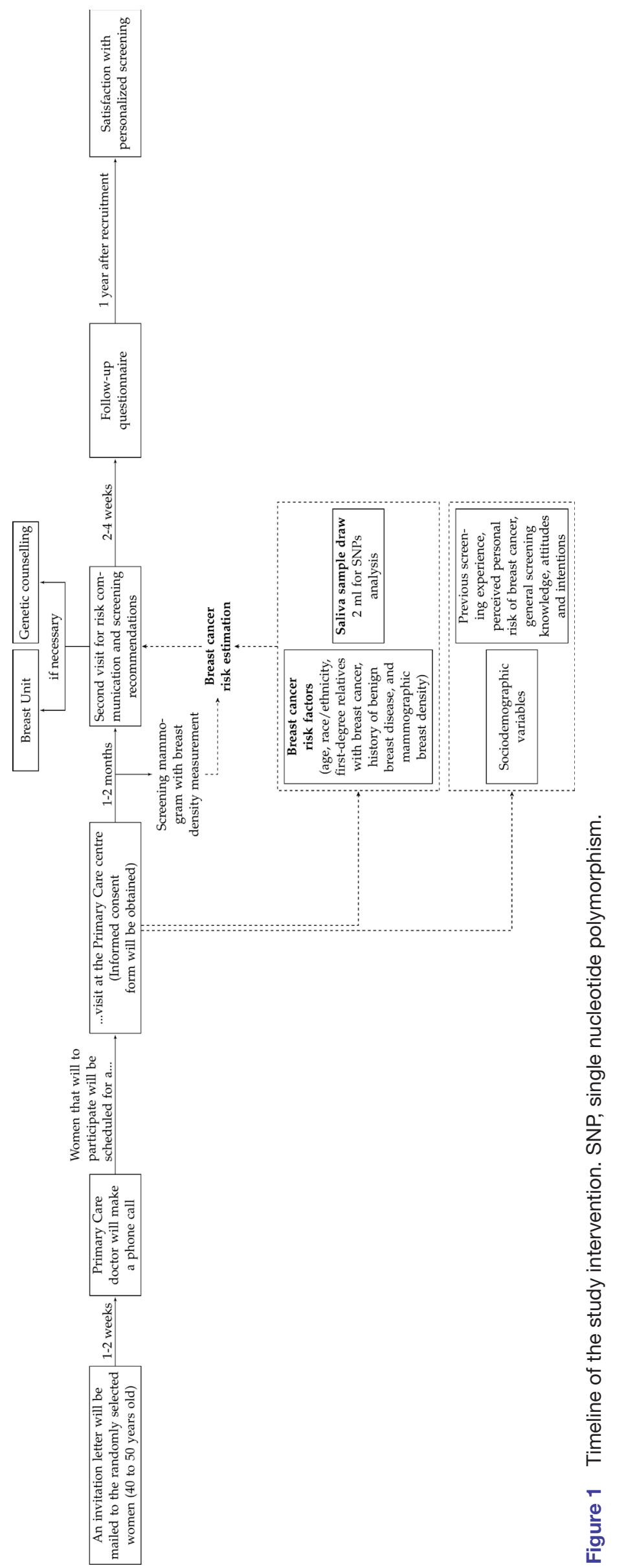


(c) and extremely dense (d). Mammographic findings will be coded from 0 (incomplete-additional imaging needed) to 6 (known biopsy-proven malignancy). Additional tests will be requested in case of abnormal results in coordination with the hospital breast unit.

Although automated breast density methods have some advantages and are common practice in research, they were not available to us. We used the clinical BI-RADS system, which is the standard of care in our healthcare centres. Even though the BI-RADS system has a nonnegligible degree of inter-reader and intra-reader variability in the categorisation of breast density, Kerlikowske et $a l^{30}$ found that automated and clinical BI-RADS density similarly predict interval and screen-detected cancer risk, suggesting that either measure may be used to inform women of their breast density. And regarding automated methods, which have shown high reproducibility and robustness, Conant $e t$ a $l^{31}$ suggest that more research and collaborative effort is needed to develop affordable, well validated and broadly available automated software.

\section{SNP determination and polygenic risk score estimation}

Collection, conservation and delivery of saliva samples will be done following the saliva collection protocol provided by the University of Lleida's Proteomics and Genomics Service (PGS) that details the procedures and indicates its storage conditions until it is delivered to the PGS. The shipment of samples from the primary care centre will be done once a week. The SNP analysis will be carried out from genomic DNA (gDNA) extracted using a suitable commercial kit. If the obtained gDNA does not meet the quality and quantity requirements necessary for the genotyping, another sample of saliva will be requested.

Genotyping of the 83 SNPs will be performed using the matrix-assisted laser desorption ionization time-offlight (MALDI-TOF) mass spectrometry technique based on the primer extension procedure where a DNA polymerase extends a primer upstream of the SNP with a set of dideoxynucleotide triphosphates (ddNTPs) on a PCR amplicon, resulting in allele-specific products for MALDI detection. ${ }^{32}$

The PRS will be obtained using the 85 SNPs associated with breast cancer that were listed in studies by Shieh $e t a l^{33}$ or Mavaddat $e t a l^{8}$ Both studies used data from the Breast Cancer Association Consortium as part of the Collaborative Oncological Gene-Environment Study.

\section{Breast cancer risk estimation}

First, the BCSC V.2.0 risk model ${ }^{5}$ and the Catalan breast cancer incidence and mortality by all causes of death will be used to estimate a preliminary 5 -year absolute risk of breast cancer. The BCSC V.2.0 model includes age, race/ethnicity, first-degree relatives with breast cancer, history of benign breast disease and mammographic breast density. The incidence data from the Girona and Tarragona cancer registries (2011 Catalan Official census, IDESCAT) will be averaged and the locally weighted smoothing (LOESS) regression will be used to smooth them by age groups. The baseline risk for the model will be obtained using the smoothed breast cancer incidence rates risk factors distribution of the BCSC dataset as described by Gail et al. ${ }^{34}$ The combination of the BCSC V.2.0 hazard ratios of the risk factors' distributions will provide the preliminary 5-year absolute risks of breast cancer.

To obtain the 5-year absolute risk that includes the PRS, the Shieh et al approach ${ }^{33}$ using the allele frequencies and odds ratios from Caucasian populations will be used. The PRS will be constructed as a composite likelihood ratio representing the individual effects of each SNP. It will be assumed that all the SNPs are inherited independently, and that there are no interactions between them. Once the PRS is obtained, a Bayesian approach will be used to update preliminary 5-year risk obtained with the BCSC V.2.0 model with the PRS value.

\section{Risk communication and screening recommendations}

Within 1-2 months after the first visit and once the breast cancer risk is obtained, a second visit will be scheduled for risk communication and screening recommendations. The visit will be performed by the same primary care doctor and team member that performed the first visit. Women will be given a report including their status for family history, benign lesions, breast density, PRS and two pictograms with the 5-year absolute risk of breast cancer, one with their risk and the other with the risk of a same age woman of the general population. The screening recommendations are presented in table 1 . Women with any anomalous or suspicious finding in mammography or with a very high risk of breast cancer after PRS assessment will be referred to the public hospital breast unit and/or genetic counselling.

It is important to remark that women aged 50 years of age and older are invited to breast cancer screening by

Table 1 Breast cancer screening recommendations according to the absolute risk of breast cancer at 5 years

\begin{tabular}{|c|c|c|}
\hline $\begin{array}{l}\text { Age group } \\
\text { (years) }\end{array}$ & $\begin{array}{l}\text { Absolute risk of } \\
\text { breast cancer at } 5 \\
\text { years }\end{array}$ & $\begin{array}{l}\text { Screening } \\
\text { recommendations }\end{array}$ \\
\hline $40-44$ & $\begin{array}{l}<0.99 \% \\
0.99 \%-1.16 \% \\
>1.16 \%\end{array}$ & $\begin{array}{l}\text { Watch and wait } \\
\text { Biennial } \\
\text { Annual }\end{array}$ \\
\hline $45-48$ & $\begin{array}{l}<0.99 \% \\
0.99 \%-1.19 \% \\
>1.19 \%\end{array}$ & $\begin{array}{l}\text { Watch and wait } \\
\text { Biennial } \\
\text { Annual }\end{array}$ \\
\hline $49-50$ & $\begin{array}{l}<0.8 \% \\
0.8 \%-1.19 \% \\
>1.19 \%\end{array}$ & $\begin{array}{l}\text { Triennial } \\
\text { Biennial } \\
\text { Annual }\end{array}$ \\
\hline $40-50$ & $>6 \%$ & $\begin{array}{l}\text { Referral to the hospital } \\
\text { breast unit and/or } \\
\text { genetic counseling }\end{array}$ \\
\hline
\end{tabular}

Absolute risk of breast cancer at 5 years for average women in Catalonia (Spain) aged 45 years: 0.8\%; 50 years: 0.99\%; 60 years: $1.16 \%$; 65 years: $1.19 \%$. 
the public system, biennially. Therefore, there is no watch and wait recommendation for women older than 49 years.

Women who receive recommendations to be screened annually or biennially and do not fulfil the age or periodicity criteria of the public screening programme are advised to inform their primary care doctors, who eventually may refer them to the radiology unit for screening mammograms at the corresponding time points.

\section{Outcomes questionnaire at follow-up}

At the end of the risk communication visit a follow-up questionnaire will be given to women. They will be asked to fill it at home and return it within 2-4 weeks. A phone call will remind women to return the questionnaire or to provide the answers by phone. The following primary and secondary outcomes will be obtained from the follow-up questionnaire.

After the study is completed, there is no plan to follow-up the participants. The study sample is too small for estimating cancer detection rates or interval cancer rates with accuracy.

\section{Sample size calculation}

Most of the primary and secondary outcomes can be expressed as proportions that will facilitate the interpretation as positive or neutral-negative outcomes. With a sample size of 385 women, proportions will be estimated with a confidence of $95 \%$ and a minimum accuracy of 0.05 . Given that the target population consists of 2038 women, a $20 \%$ participation acceptance would provide the needed sample size.

\section{Participant timeline}

The study started to include participants on 11 December 2018. On 13 March 2019 participant number 252 was included and the next day accrual was suspended because of the COVID-19 pandemic. On 21 October 2020, the study enrolment resumed. We estimate that three additional months of accrual are needed. The participant timeline is detailed in figure 1 .

\section{Outcomes}

\section{Primary outcomes}

The primary outcome measures will be attitude towards, intention to participate in and satisfaction with personalised breast cancer screening by participant women.

\section{Attitude towards personalised breast cancer screening}

It will be measured with a scale with three items adapted from Hersch et al..$^{35}$ Each item ranges from 1 to 5, with a total score ranging from 3 to 15 . One of the items asks participants their opinion on varying the frequency of screening exams depending on breast cancer risk. The other two items ask if participants would be satisfied to be invited more/less frequently in case that they had a higher/lower risk of breast cancer than the average women. Higher scores indicate more positive attitudes. A 'positive attitude' is defined as a total score $\geq 12$.
Intention to participate in personalised breast cancer screening It will be measured with a 5-point Likert scale from definitely will (1) to definitely will not (5), adapted from Hersch et $a l^{35}$ The variable will be dichotomised as intending to participate (definitely or likely) or not.

\section{Satisfaction with personalised screening}

It will be measured in a 5-point Likert scale from not at all satisfied (1) to extremely satisfied (5). ${ }^{536}$ This variable will be measured after 1 year of recruitment.

\section{Secondary outcomes}

Attitudes towards screening mammography

It will be measured using five items adapted from Hersch et $a l^{35}$ Each item ranges from 1 to 5 . Total scores can range from 5 to 25 . A positive attitude is defined as a total score $\geq 20$. Higher scores indicate more positive attitudes.

\section{Attitude towards measuring breast cancer risk}

One categorical variable with four categories. It asks if the measure of breast cancer risk will do: more harm than good, more good than harm, it depends, do not know. The absolute and relative frequencies of the four categories will be obtained. $^{36}$

\section{Emotional impact of the measure of breast cancer risk}

Three categorical variables with five categories, ranging from strongly disagree (1) to strongly agree (5). First variable: the information about the individual risk of breast cancer provides calmness. Second variable: receiving information about risks produces anxiety. Third variable: the information about the individual risk of breast cancer makes me worry. The scores of the three items will not be added as a scale, they will be reported separately. ${ }^{35}$

\section{Preference with regard to the current screening}

Categorical variable with three categories. It asks what type of screening the participants would choose, personalised risk-based or 'one-size-fits-all,' which is biennial between 50 and 69 years in Spain.

\section{Knowledge of the benefits and harms of breast cancer screening}

Eleven conceptual knowledge questions (yes/no) and four numerical knowledge questions with categories on the effect of screening. A total of 22 marks could be obtained, 11 coming from the questions on conceptual knowledge and 11 coming from the questions on numerical knowledge that measured absolute and relative values of the screening outcomes. The threshold to define adequate knowledge is to score at least $50 \%$ of the available marks, including at least one numerical mark, on all the three screening outcome subscales that refer to mortality reduction, overdiagnosis and false positives. ${ }^{35} 37$

\section{Decisional conflict}

O'Connor Decisional Conflict Scale, 10-item low literacy version, on a scale from 0 (no decisional conflict) to 100 (extreme decisional conflict). ${ }^{38}$ Scores $<25$ are associated with implementing decisions; scores exceeding 37.5 are 
associated with decision delay or feeling unsure about implementation.

\section{Confidence in the decision}

Three Likert scale statements rated from 1 (not at all confident) to 5 (very confident). A total score is obtained summing the scores of the three items and dividing by three. ${ }^{39}$

\section{Anxiety about screening participation}

Six-item short form of the Spielberger State Trait Anxiety Inventory (STAI) on a scale from 20 to 80, with higher scores indicating greater levels of anxiety. ${ }^{40}$ To calculate the total STAI score, each item is rated from 1 (not at all) to 4 (very much so), scoring of the positive items (calm, relaxed, content) is reversed, all six scores are summed and the total score is multiplied by $20 / 6$.

Perceived significance of the benefits and the adverse effects of screening

Women will be asked how important it is for them to consider the chances of (1) avoiding breast cancer death, (2) being diagnosed and treated for a cancer that is not harmful and (3) having a false positive. The response options range from very important (1) to not at all important (4). The scores of the three items will not be added as a scale, they will be reported separately. ${ }^{35}$

\section{Self-efficacy}

Four items ranging from strongly disagree (1) to strongly agree (5). Total scores can range from 4 to 20. Higher scores indicate higher self-efficacy. ${ }^{36}$

\section{Experience assessment}

It will be measured using five items. Each item ranges from 1 to 5 . Total scores can range from 5 to 25. A positive assessment is defined as a total score $\geq 20$. Higher scores indicate more positive experience assessment. ${ }^{36}$

\section{Confidence in personalised screening}

Confidence Likert scale with one item ranging from very low confidence (1) to very high confidence (5). ${ }^{36}$

\section{Understanding of the individual risk and the screening recommendations}

Two categorical variables with five categories each, ranging from strongly disagree (1) to strongly agree (5). First variable: I understood the information I received about my risk of breast cancer in relation to women of my age. Second variable: I have understood the recommendations given to me about the screening of breast cancer in the coming years, based on my risk of breast cancer. The scores of the two items will be reported separately. ${ }^{36}$

\section{Time spent on risk communication}

Continuous variable, number of minutes. Recorded by the participant doctors.
Proportion of women who accept to participate in the study Number of women that accept to participate divided by number of women contacted.

Proportion of participating women who complete the different phases of the study

Number of women that complete the different phases divided by number of participating women.

\section{Data management}

The data will be collected and analysed by the DECIDO research team. The data will be stored securely in password-protected computer files and in locked cabinets at the Lleida Institute for Biomedical Research (IRBLleida). Access to these files will be granted only to the research team.

\section{Data analysis}

Primary and secondary outcomes will be described with means and SD or medians and quartiles. For scale variables, individual items, subscales and overall scores will be presented. Proportions of positive responses, such as positive attitude or adequate knowledge, will be obtained as stated in the outcomes definitions.

The $95 \%$ CIs for proportions will be obtained using the exact binomial distribution. For means, the $95 \%$ CIs will be obtained assuming that the sample mean distribution follows the Student's t-test distribution with $\mathrm{n}-1 \mathrm{df}$.

As a descriptive analysis, primary and secondary outcomes by level of knowledge of the benefits and harms of screening and by the age groups and screening recommendations shown in table 1 , will be presented.

The $\mathrm{R}$ programming language ${ }^{41}$ and the RStudio environment ${ }^{42}$ will be used for the data analysis.

\section{Patient and public involvement}

Participants were not and will not be involved in the research process.

\section{ETHICS AND DISSEMINATION}

The study protocol was approved by the Drug Research Ethics Committee of the University Hospital Arnau de Vilanova. The trial will be conducted in compliance with this study protocol, the Declaration of Helsinki and Good Clinical Practice.

The results will be published in peer-reviewed scientific journals, and locally and internationally disseminated in scientific conferences and media.

\section{Author affiliations}

${ }^{1}$ Eixample Basic Health Area, Catalan Institute of Health, Lleida, Spain

${ }^{2}$ Health PhD Program, University of Lleida, Lleida, Spain

${ }^{3}$ Basic Medical Sciences, University of Lleida, Lleida, Spain

${ }^{4}$ Research Group on Statistics and Economic Evaluation in Health (GRAEES), University of Lleida, Lleida, Spain

${ }^{5}$ Department of Computing and Industrial Engineering, University of Lleida, Lleida, Spain

${ }^{6}$ Primer de Maig Basic Health Area, Catalan Institute of Health, Lleida, Spain

${ }^{7}$ Proteomics and Genomics Service, University of Lleida, Lleida, Spain

${ }^{8}$ Radiology Department, Arnau de Vilanova University Hospital, Lleida, Spain 
${ }^{9}$ Breast Cancer Screening Program, Catalan Institute of Health, Lleida, Spain ${ }^{10}$ IRBLleida, Lleida, Spain

Correction notice This article has been corrected since it was first published. The table 1 has been updated. The provenance and peer review statement has been included.

Acknowledgements The authors would like to thank the Oncolliga Lleida organisation for their commitment to the DECIDO project. The authors would also like to thank JP Glutting for review and editing.

Collaborators The DECIDO Group: Pau Balaguer-Llaquet; Iván-David Benítez; Alexandra Bertran; Àngels Cardona; Misericòrdia Carles-Lavila; Cristina CazorlaSánchez; Núria Codern; Inés Cruz-Esteve; Carles Forné-Izquierdo; Maria José Hernández-Andreu; Edelmir Iglesias; Gisela Galindo-Ortego; Marta Hernández; Celmira Laza-Vásquez; Montserrat Llorens-Gabandé; Montserrat Martínez-Alonso; Maria José Pérez-Lacasta; Hèctor Perpiñán; Anna Pons-Rodríguez; Montserrat Rué; Isabel Sánchez-López; Jordi Vilaplana-Mayoral; Mercè Reñé-Reñé.

Contributors Conception and design of the study: MR, CFI, MM-A. Clinical visits, early detection exams and acquisition of the data: IC-E, MR-R, CC, MH-A, GG-0, MLG, CL-V, AP-R. Genomics analyses: IS-E. Information system design and elaboration: JV-M, PB-L. Risk models adaptation and statistical analysis plan: $\mathrm{CFI}$ MM-A, MR. Initial draft of the manuscript: AP-R, MM-A, MR. Critical revision: CFI, IS-L, IC-E, GG-0, MH-A, CC, CL-V. All authors critically revised the manuscript for intellectual content and approved the final version.

Funding This study was supported by the research grant 'Personalized breast cancer screening: assessment of its feasibility and acceptability in the National Health System' (PI17/00834) from the Instituto de Salud Carlos III and cofunded by Fondo Europeo de Desarrollo Regional (FEDER) 'Una manera de hacer Europa'. Celmira Laza Vásquez received a grant from Santander Program scholarship 2020 as a predoctoral fellow at the University of Lleida. The non-profit organisation Oncolliga Lleida (grant number N/A) will fund part of the genomic analyses for breast cancer risk assessment.

Competing interests None declared.

Patient and public involvement Patients and/or the public were not involved in the design, or conduct, or reporting, or dissemination plans of this research.

Patient consent for publication Not required.

Provenance and peer review Not commissioned; externally peer reviewed.

Open access This is an open access article distributed in accordance with the Creative Commons Attribution Non Commercial (CC BY-NC 4.0) license, which permits others to distribute, remix, adapt, build upon this work non-commercially, and license their derivative works on different terms, provided the original work is properly cited, appropriate credit is given, any changes made indicated, and the use is non-commercial. See: http://creativecommons.org/licenses/by-nc/4.0/.

ORCID iDs

Carles Forné Izquierdo http://orcid.org/0000-0002-8133-3274

Montserrat Rué http://orcid.org/0000-0002-7862-9365

\section{REFERENCES}

1 European Commission. European Commission 2015/C421/01 Non-opposition to a notified concentration. Official Journal of the European Union 2015.

2 Chowdhury S, Dent T, Pashayan N, et al. Incorporating genomics into breast and prostate cancer screening: assessing the implications. Genet Med 2013;15:423-32.

3 Vilaprinyo E, Forné C, Carles M, et al. Cost-Effectiveness and harmbenefit analyses of risk-based screening strategies for breast cancer PLoS One 2014;9:e86858.

4 Tice JA, Cummings SR, Smith-Bindman R, et al. Using clinical factors and mammographic breast density to estimate breast cancer risk: development and validation of a new predictive model. Ann Intern Med 2008;148:337-47.

5 Tice JA, Miglioretti DL, Li C-S, et al. Breast density and benign breast disease: risk assessment to identify women at high risk of breast cancer. J Clin Oncol 2015;33:3137-43.

6 Shieh Y, Eklund M, Madlensky L, et al. Breast cancer screening in the precision medicine era: risk-based screening in a population-based trial. J Natl Cancer Inst 2017;109:djw290.
7 Michailidou K, Lindström S, Dennis J, et al. Association analysis identifies 65 new breast cancer risk loci. Nature 2017;551:92-4.

8 Mavaddat N, Pharoah PDP, Michailidou K, et al. Prediction of breast cancer risk based on profiling with common genetic variants. J Natl Cancer Inst 2015;107:djv036.

9 Vachon CM, Pankratz VS, Scott CG, et al. The contributions of breast density and common genetic variation to breast cancer risk. J Natl Cancer Inst 2015;107:1-4.

10 Vachon CM, Scott CG, Tamimi RM, et al. Joint association of mammographic density adjusted for age and body mass index and polygenic risk score with breast cancer risk. Breast Cancer Res 2019;21:1-10.

11 Yanes T, Young M-A, Meiser B, et al. Clinical applications of polygenic breast cancer risk: a critical review and perspectives of an emerging field. Breast Cancer Res 2020;22:1-10.

12 Shieh Y, Hu D, Ma L, et al. Joint relative risks for estrogen receptorpositive breast cancer from a clinical model, polygenic risk score, and sex hormones. Breast Cancer Res Treat 2017;166:603-12.

13 Román M, Sala M, Domingo L, et al. Personalized breast cancer screening strategies: a systematic review and quality assessment. PLoS One 2019;14:e0226352.

14 Evans DGR, Donnelly LS, Harkness EF, et al. Breast cancer risk feedback to women in the UK NHS breast screening population. $\mathrm{Br} J$ Cancer 2016;114:1045-52.

15 Tyrer J, Duffy SW, Cuzick J. A breast cancer prediction model incorporating familial and personal risk factors. Stat Med 2004;23:1111-30.

16 Evans DGR, Harkness EF, Brentnall AR, et al. Breast cancer pathology and stage are better predicted by risk stratification models that include mammographic density and common genetic variants. Breast Cancer Res Treat 2019;176:141-8.

17 French DP, Astley S, Brentnall AR, et al. What are the benefits and harms of risk stratified screening as part of the NHS breast screening programme? study protocol for a multi-site non-randomised comparison of BC-predict versus usual screening (NCT04359420). BMC Cancer 2020;20:1-14.

18 Pérez-Lacasta MJ, Sala M, Perestelo-Pérez L, et al. Effect of information about the benefits and harms of mammography on women 's decision making : The InforMa randomised controlled trial. PLoS One 2019:1-20.

19 Puig-Vives M, Pollan M, Rue M, et al. Rapid increase in incidence of breast ductal carcinoma in situ in Girona, Spain 1983-2007. Breast 2012;21:646-51.

20 Martinez-Alonso M, Vilaprinyo E, Marcos-Gragera R, et al. Breast cancer incidence and overdiagnosis in Catalonia (Spain). Breast Cancer Res 2010;12:R58.

21 Rainey L, Jervaeus A, Donnelly LS, et al. Women's perceptions of personalized risk-based breast cancer screening and prevention: An international focus group study. Psychooncology 2019;28:1056-62.

22 Koitsalu M, Sprangers MAG, Eklund M, et al. Public interest in and acceptability of the prospect of risk-stratified screening for breast and prostate cancer. Acta Oncol 2016;55:45-51.

23 Rainey L, van der Waal D, Donnelly LS, et al. Women's decisionmaking regarding risk-stratified breast cancer screening and prevention from the perspective of international healthcare professionals. PLoS One 2018;13:e0197772.

24 Puzhko S, Gagnon J, Simard J, et al. Health professionals' perspectives on breast cancer risk stratification: understanding evaluation of risk versus screening for disease. Public Health Rev 2019;40:1-19.

25 Esquivel-Sada D, Lévesque E, Hagan J, et al. Envisioning implementation of a personalized approach in breast cancer screening programs: stakeholder perspectives. Healthc Policy 2019;15:39-54.

26 Hoffmann TC, Del Mar C. Patients' expectations of the benefits and harms of treatments, screening, and tests: a systematic review. JAMA Intern Med 2015;175:274.

27 Hoffmann TC, Del Mar C. Clinicians' expectations of the benefits and harms of treatments, screening, and tests: a systematic review. JAMA Intern Med 2017;177:407-19.

28 Toledo-Chávarri A, Rué M, Codern-Bové N, et al. A qualitative study on a decision aid for breast cancer screening: views from women and health professionals. Eur J Cancer Care 2017;26:e12660-11.

29 American College of Radiology. Breast imaging reporting and data system (BI-RADS. 5th ed, 2013.

30 Kerlikowske K, Scott CG, Mahmoudzadeh AP, et al. Automated and clinical breast imaging reporting and data system density measures predict risk for screen-detected and interval cancers: a case-control study. Ann Intern Med 2018;168:757-65. 
31 Conant EF, Sprague BL, Kontos D. Beyond BI-RADS density: a call for quantification in the breast imaging clinic. Radiology 2018;286:401-4.

32 Sauer S, Gut IG. Genotyping single-nucleotide polymorphisms by matrix-assisted laser-desorption/ionization time-of-flight mass spectrometry. J Chromatogr B Analyt Technol Biomed Life Sci 2002;782:73-87.

33 Shieh Y, Hu D, Ma L, et al. Breast cancer risk prediction using a clinical risk model and polygenic risk score. Breast Cancer Res Treat 2016;159:513-25.

34 Gail MH, Brinton LA, Byar DP, et al. Projecting individualized probabilities of developing breast cancer for white females who are being examined annually. J Natl Cancer Inst 1989;81:1879-86.

35 Hersch J, Barratt A, Jansen J, et al. Use of a decision aid including information on overdetection to support informed choice about breast cancer screening: a randomised controlled trial. Lancet 2015;385:1642-52.

36 Sekhon M, Cartwright M, Francis JJ. Acceptability of healthcare interventions: an overview of reviews and development of a theoretical framework. BMC Health Serv Res 2017;17:1-13.

37 Carles M, Martínez-Alonso M, Pons A, et al. The effect of information about the benefits and harms of mammography on women's decision-making: study protocol for a randomized controlled trial. Trials 2017;18:1-8.

38 O'Connor AM. Decisional conflict Scale-user manual 1993. decision aid evaluation measures, 2010. Available: http://decisionaid.ohri.ca/ eval_dcs.html;

39 O'Connor AM. Decision self-efficacy Scale-user manual 1995. decision aid evaluation measures, 2002. Available: http://decisionaid. ohri.ca/eval_self.html;

40 Marteau TM, Bekker $\mathrm{H}$. The development of a six-item short-form of the state scale of the Spielberger State-Trait anxiety inventory (STAI). Br J Clin Psychol 1992;31:301-6.

41 R Core Team. R: a language and environment for statistical computing, 2020. Available: https://www.R-project.org/

42 RStudio Team. RStudio: integrated development environment for R, 2020. Available: http://www.rstudio.com/ 